\title{
Numerical Investigation in Energy Shaping Control of Inverted Flexible Pendulum
}

\author{
C. Kirankumar and Dr. S. Periyasamy
}

\begin{abstract}
The reciprocating mechanism is subjected to various forces. The resultant of the considerable number of force following up on the body of the instrument because of dormancy force just is known as shaking force. In this way if the resultant of the considerable number of forces because of unbalanced impacts is zero, at that point there will be an uneven force, so unbalanced force is created in mechanical system. So this type problem occurs in locomotives and reciprocating mechanical systems. So the additional parts like pendulum springs are included in mechanical system to minimize the unbalanced forces or couples. The impact of an uneven essential force perpendicular to the line of stroke is to create variety in pressure on the rails, which results in hammering action on the rails. In this project inverted flexible pendulum is used to minimize the vibration using the function.

Existing system is adapted with Laplace equation of damping factor. From that the data like velocity, acceleration, and force and swing angle were calculated from the modified system. So, the angle of swing and forces are varying with time was changed and the vibration response was measured. This inverted pendulum may be used in automatic / guided vehicle operated in a reciprocating mechanism.
\end{abstract}

Index Terms - pendulum, swing angle, AGV, displacement

\section{INTRODUCTION}

A reversed pendulum has that focal point of mass over its rotate point (M.S.Alamand ,M.O.Tokhi, 2007). It is temperamental and without extra assist will with falling over. It very well may be suspended stable right now by utilizing a control system to monitor the edge of the post and move the turning point on a level plane back under the focus point of mass when it begins to fall over, keeping it adjusted (Bo Luo,2013). The modified pendulum is exemplary in elements and control dynamics and is utilized as a benchmark for testing control methodologies the adjustment of under activated mechanical system, both in the area of customary and incomplete differential conditions, has been widelyaddressed by several control researchers in recent years (Mohammad H. Fateh 2019).

Manuscript revised on February 29, 2020 and published on March 10, 2020

C.Kirankumar, M.E - Engineering Design, Anna University/ Government College of Technology/ Coimbatore - 641013, India.
In the domain of flexible mechanisms and robots, flexibility in the links is the main source of under actuation. In the event that the mishappening because of adaptability are little, it is conceivable to utilize an unconstrained

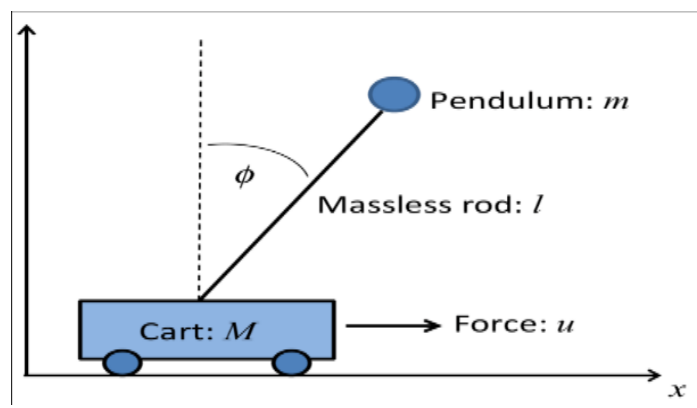

Figure 1. Inverted pendulum

Lagrange definition and conjure the Assumed Modes Method (AMM) to acquire a straightforward, limited dimensional model. This displaying strategy, in any case, in pertinent for the mechanical system with enormous distortions, for which a compelled Euler-Lagrange (EL) detailing is required. Potential vitality change inferable from ultra-enormous disfigurements within the sight of gravity is considered (Juqian Zhang, BangchunWen, Xueliang Zhang and YunshanLiu,2019). Utilizing the steady length of the bar as a holonomic imperative. The plan depends on a model, got by means of the compelled Lagrange definition. The controller configuration comprises of a halfway criticism linearization step kept by a standard PID (proportional integral derivative) controller following up on two inactive yields (J. Siang, M.H. 2018). Boundedness everything being equal and (nearby) asymptotic security of the ideal harmony is hypothetically settled and theoretical established.

\section{A. Objectives of Inverted Flexible Pendulum}

To stabilize the unstable cart-pendulum system simultaneously physical constraints imposed. To identify the non-linear cart friction that will be helpful in reducing the modeling error and it will decrease the stick-slip oscillations (friction) (X. Zhao 2015). To reduce the vibration and control the oscillation. To control the unstable position is stabilized and at the same time the cart is driven to a desired position. To calculate the velocity, acceleration and force. 


\section{B. Application of Inverted Flexible Pendulum}

Automobile parts in vehicle, Robotics, Structural members, Missile launching system, Balancing systems, Human walking

\section{Controlling Techniques}

Inverted flexible pendulum in automated guided vehicle to reduce the vibration control. The control issue in these mechanical systems is testing, particularly with gravity coupling and huge deformation, due to natural under incitation and the mix of lumped and dispersed parameters of nonlinear mechanical systems (V. Casanova, J. Alcaína, J. 2015). Move the payload to an ideal area and diminishing the payload swing, by a nonlinear controller for moderate elements. Suppress transverse vibrations of the link, by a direct controller for quick elements. Controlling an inverted pendulum is a fundamental of stabilizing unstable systems such as walking robots. Controlling an inverted pendulum is a fundamental of stabilizing unstable systems such as walking robots. The controlled system made up of a wire-driven bearer, an adaptable beam pivoted to the Carrier, and a weight fixed on the opposite side of the beam.

\section{Mathematical Modelling OF INVERTED Flexible PENDULUM}

The following dimensions were used to model the inverted pendulum

\begin{tabular}{|l|l|l|l|}
\hline \multicolumn{1}{|c|}{ Table No 1 Dimensions Of Inverted Pendulum } \\
\hline Parameter & $\begin{array}{l}\text { Symbo } \\
\text { I }\end{array}$ & Value & Units \\
\hline $\begin{array}{l}\text { Pendulum cross } \\
\text { section area }\end{array}$ & $\mathrm{A}$ & $7 \times 10^{-6}$ & $\mathrm{~m} 2$ \\
\hline $\begin{array}{l}\text { Young's modulus } \\
\text { Gravitational } \\
\text { acceleration }\end{array}$ & $\mathrm{E}$ & $8 \times 10^{10}$ & $\mathrm{~N} / \mathrm{m} 2$ \\
\hline $\begin{array}{l}\text { Moment of inertia } \\
\mathrm{m} / \mathrm{sec} 2\end{array}$ & $\mathrm{I}$ & $\begin{array}{l}1.056 \times \\
10^{-13}\end{array}$ & $\begin{array}{l}\mathrm{kg} \\
\mathrm{m} 2\end{array}$ \\
\hline $\begin{array}{l}\text { Pendulum length } \\
\text { Function of the } \\
\text { system natural } \\
\text { frequency }\end{array}$ & $\mathrm{L}$ & 0.305 & $\mathrm{~m}$ \\
\hline $\begin{array}{l}\text { Dimensionless } \\
\text { constant }\end{array}$ & $\gamma$ & 1.1741 & - \\
\hline $\begin{array}{l}\text { Pendulum density } \\
\text { Pendum }\end{array}$ & $\rho$ & 8400 & $\mathrm{Kg} / \mathrm{m} 3$ \\
\hline
\end{tabular}

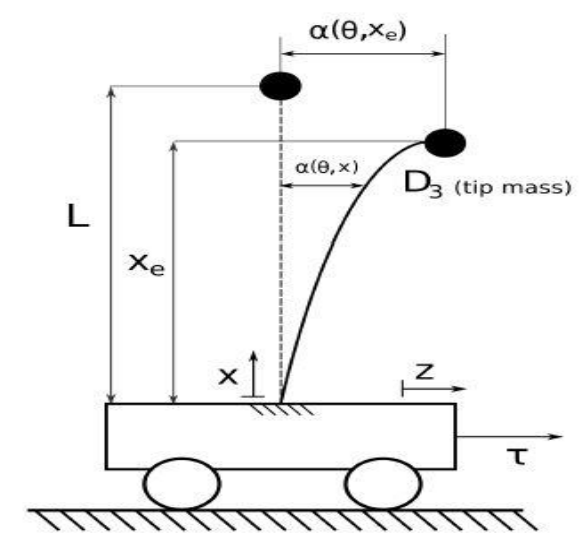

Figure 2 Single ultra-flexible link with base excitation

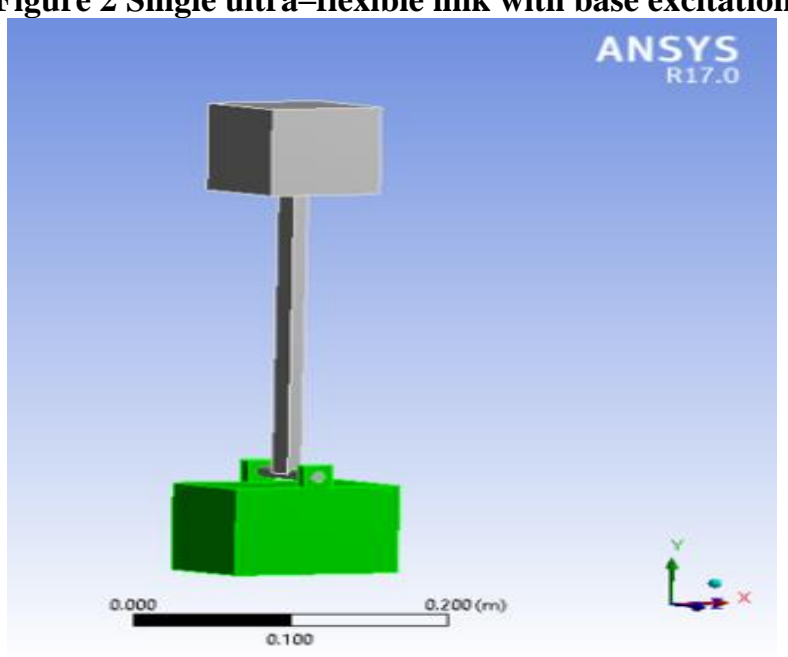

Figure 3 model of Inverted type pendulum

\section{A. Command Shaping for Vibration Control}

A vibratory system can be demonstrated as a superposition of second-order differential equation with a transfer function

$$
G(s)=\frac{w_{n}^{2}}{s^{2}+2 \xi w_{n} s+w_{n}^{2}}
$$

Where, $w_{n}$ is the natural frequency and $\zeta$ is the damping ratio of the system. Thus, the impulse response of the system at time $t$ is:

$$
y(t)=\frac{A w_{n}}{\sqrt{1-\xi^{2}}} e^{-\xi w_{n}\left(t-t_{0}\right) \sin \left[w_{n} \sqrt{1-\xi^{2}}\left(t-t_{0}\right)\right]}
$$

Where, $\mathrm{A}$ and $t_{0}$ are the amplitude and time-location of the impulse, respectively. For $\mathrm{N} \quad \omega_{\mathrm{n}}=$ impulse the impulse response can be expressed as;

Where

$$
\begin{gathered}
M=\sqrt{\left(\sum_{i=1}^{N} B_{i} \cos \phi_{i}\right)^{2}+\left(\sum_{i=1}^{N} B_{i} \sin \phi_{i}\right)^{2}} \\
B_{i}=\frac{A w_{n}}{\sqrt{1-\xi^{2}}} e^{-\xi w_{n}\left(t_{n}-t_{i}\right)} \\
\phi_{i}=w_{d} t_{i}
\end{gathered}
$$




$$
\alpha=\tan ^{-1}\left(\sum_{i=1}^{N} \frac{B_{i} \cos \phi_{i}}{B_{i} \sin \phi_{i}}\right)
$$

And $\mathrm{Ai}$ and ti are the magnitudes of the impulses and their time-locations, respectively.

Where,

$$
V=\sqrt{V_{1}^{2}+V_{2}^{2}}
$$

$$
V_{1}=\sum_{i=1}^{N} \frac{A w_{n}}{\sqrt{1-\xi^{2}}} e^{-\xi w_{n}\left(t_{n}-t_{i}\right) \cos \left(w_{d} t_{i}\right)}
$$

To accomplish zero vibration after the last impulse, it is necessitated that both $\mathrm{V}_{1}$ and $\mathrm{V}_{2}$ in equation are independently zero. Moreover, to guarantee that the molded

$$
V_{2}=\sum_{i=1}^{N} \frac{A w_{n}}{\sqrt{1-\xi^{2}}} e^{-\xi w_{n}\left(t_{n}-t_{i}\right) \sin \left(w_{d} t_{i}\right)}
$$

Direction input creates the equivalent unbending body movement as the unshaped order, it is necessitated that the total of amplitudes of the driving forces is solidarity. To avoid from reaction delay, the first impulse is selected at time $t_{1}=0$. Hence, setting $V_{1}$ and $V_{2}$ in equation to zero and For zero vibration $(\mathrm{ZV})$, the time locations and amplitudes of impulses are given as

$$
\begin{gathered}
t_{1}=0 \\
t_{2}=\frac{\pi}{w_{d}} \\
t_{3}=\frac{3 \pi}{w_{d}} \\
A_{1}=\frac{1}{1+2 k+k^{2}} \\
A_{2}=\frac{2 k}{1+2 k+k^{2}} \\
A_{3}=\frac{k^{2}}{1+2 k+k^{2}} \\
K=e \frac{-\frac{\xi \pi}{\sqrt{1-\xi^{2}}}}{w_{d}=w_{n} \sqrt{1-\xi^{2}}}
\end{gathered}
$$

Where, $\omega_{\mathrm{n}}$ is the natural frequency and $\zeta$ is the damping ratio of the system. For the forming procedure to be viable on a genuine system, the shaper must have heartiness to displaying mistakes. One sort of strong shaper can be acquired by requiring the subsidiary as for the recurrence of the remaining vibration to be equivalent to zero. The time locations and amplitudes of ZVD (zero vibration derivative)based CS are as:

$$
y(t)=M \sin \left(w_{d} t+\alpha\right)
$$

Unlike the ZV and ZVD shapers, the extra insensitive (EI) shaper doesn't endeavor to drive the vibration to zero at the displaying frequency. Or maybe, the vibration is restricted to some low, however adequate degree of remaining vibration. The time locations and amplitudes of EI-based CS are as

$$
\begin{aligned}
t_{1} & =0 \\
t_{2} & =\frac{\pi}{w_{d}} \\
t_{3} & =\frac{2 \pi}{w_{d}}
\end{aligned}
$$

$$
\begin{gathered}
t_{4}=\frac{3 \pi}{w_{d}} \\
A_{1}=\frac{1}{1+3 k+3 k^{2}+k^{3}} \\
A_{2}=\frac{3 k}{1+3 k+3 k^{2}+k^{3}} \\
A_{3}=\frac{3 k^{2}}{1+3 k+3 k^{2}+k^{3}} \\
A_{4}=\frac{k^{3}}{1+3 k+3 k^{2}+k^{3}}
\end{gathered}
$$

\section{B. Swing Angle of The Flexible Pendulum}

A pendulum is a bob suspended from a rotate about the pivot point. Clearly, a true pendulum would live in a $3 \mathrm{D}$ space, yet taking less difficult situations, a pendulum in a 2D space the program canvas ([6]X. Yang, X. Zheng,).
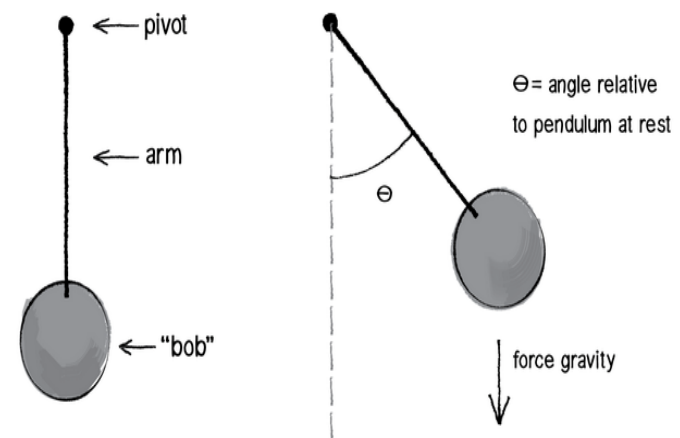

Figure 4 swing angle of the flexible pendulum $(\theta)$

In the Forces section, come to know that such as the force of gravity shown in the diagram above causes an object to accelerate $\mathrm{F}=$ ma.

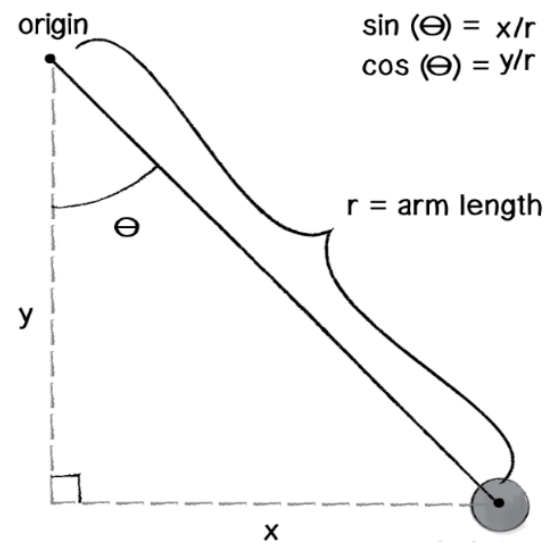

Figure 5 swing angle make with coordinate ( $\theta$ swing angle)

Assuming the $\theta$ (swing angle) as trigonometry coordinates $(\mathrm{x}, \mathrm{y})$. So comparative with the origin, the pendulum's position is a polar coordinate $(r, \theta)$. And should converted to be Cartesian coordinate. In that section, the angle was relative to the horizontal axis, but here, it's relative to the vertical axis, so it will end up using $\sin (\theta)$ for theses position and $\cos (\theta)$ for the $y$ position, instead of $\cos (\theta)$ and $\sin (\theta)$, respectively. Using the conversion formula, the position comparative with the origin can be calculated. 


\section{UNITS}

The inverted flexible pendulum has been located in various mechanisms. Hence forth, sets of readings have been taken for the movement of pendulum along the path automated guided vehicle. Here the proposed pendulum model was reduce the vibration noise of automated guided vehicle. From this velocity, acceleration, displacement, forces were taken for analysis.

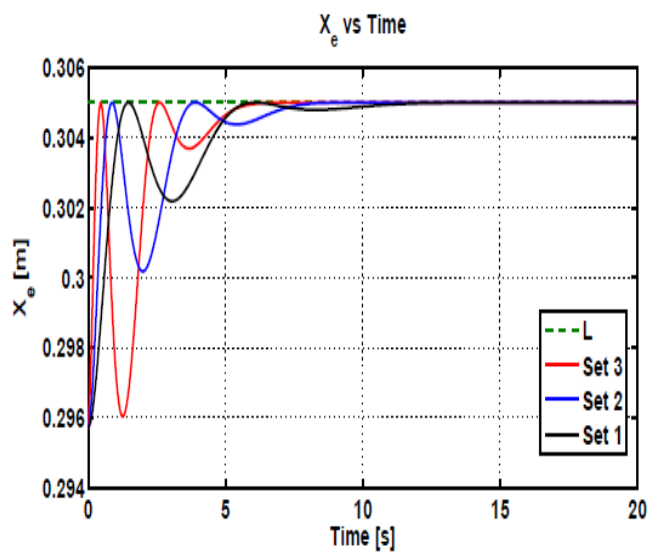

Figure 6 Displacement Vs time

Figure 6 the $\mathrm{x}$-axis taken for time and $\mathrm{y}$ axis for displacement. Here the existing method peak value is 0.296 and proposed model displacement value is 0.20 was taken from this graph. These relations can be comprehended by studying the above graph.

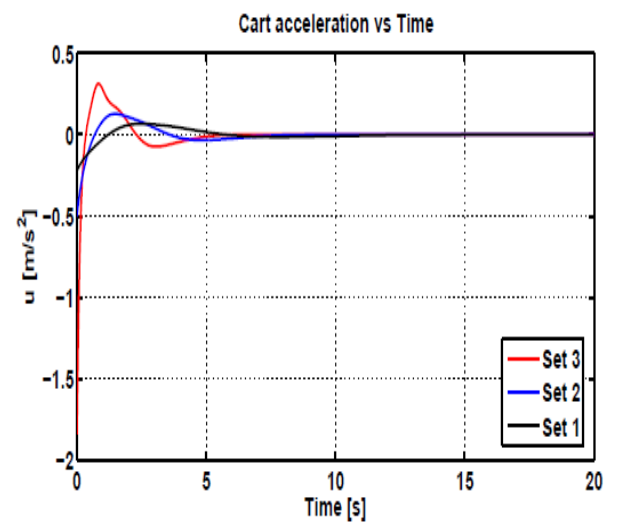

Figure 7 Acceleration Vs time

Figure 7 the $\mathrm{x}$-axis taken for time and $\mathrm{y}$ axis for acceleration. Here the existing method peak value is 0.4 and proposed model displacement value is 0.20 was taken from this graph. These relations can be comprehended by studying the above graph.

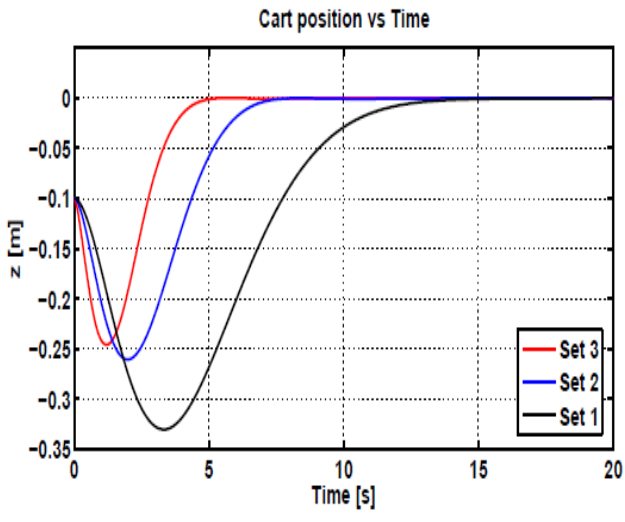

Figure 8 Displacement 1Vs time

Figure 8 the $\mathrm{x}$-axis taken for time and y axis for displacement. Here the existing method peak value is -0.27 and proposed model displacement value is -0.23 was taken from this graph. These relations can be comprehended by studying the above graph.

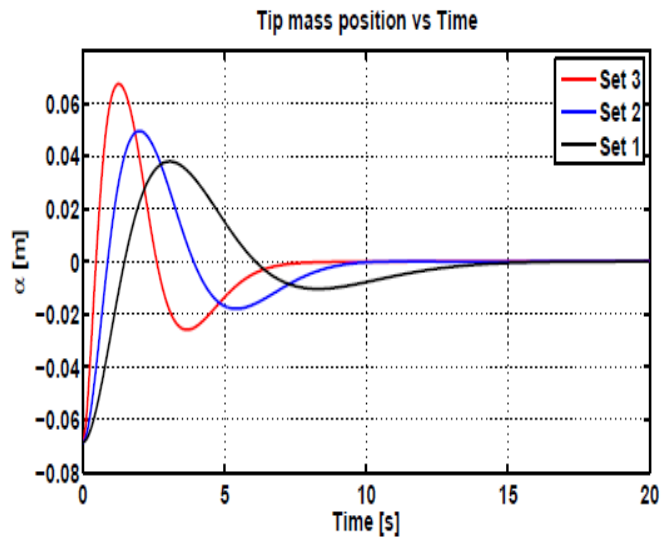

Figure 9 Amplitude Vs time

Figure 9 the $\mathrm{x}$-axis taken for time and $\mathrm{y}$ axis for displacement. Here the existing method peak value is 0.05 and proposed model displacement value is 0.06 was taken from this graph within 5 seconds the amplitude was reduced. These relations can be comprehended by studying the above graph.

Figure 9 Applied force for moving the AGV system when applying different forces

Figure 9 the $\mathrm{x}$-axis taken for time and y axis for force. Here the existing method peak value is 12 and proposed model displacement value is 8 was taken from this graph. These relations can be comprehended by studying the above graph.

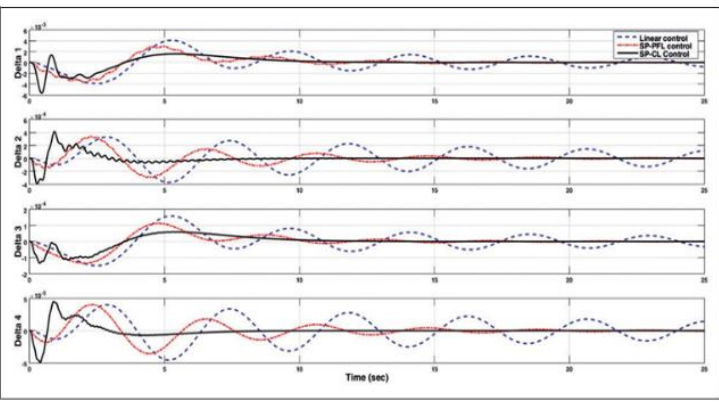

Figure 10 The amplitudes of the first four vibration modes of the AGV when applying different forces 
Figure 10 The $\mathrm{x}$-axis taken for time and $\mathrm{y}$ axis for different forces. Here the existing method peak value is 4 and proposed model displacement value is 2.5 was taken from this graph. And also the three set of nodes are plotted from this graph. These relations can be comprehended by studying the above graph.

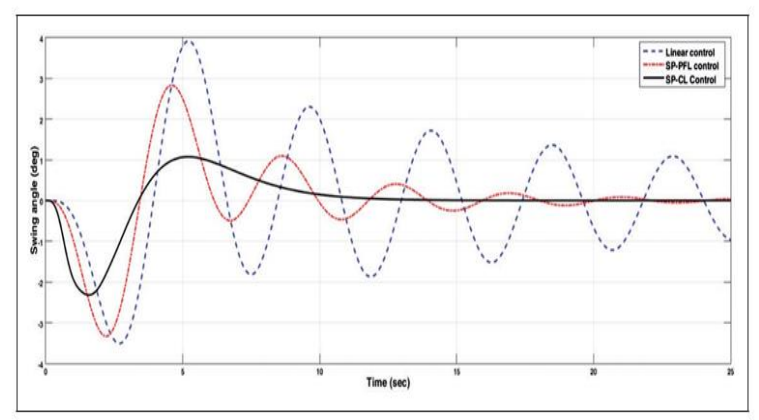

Figure 11 AGV system when applying force swing angle during the traveling of the path

Figure 11 The $\mathrm{x}$-axis taken for time and $\mathrm{y}$ axis for swing angle. Here the existing method peak value is 4 and proposed model displacement value is 2.5 was taken from this graph. And also the three set of nodes are plotted from this graph. These relations can be comprehended by studying the above graph.

8 Different position vs torque condition and velocity 1

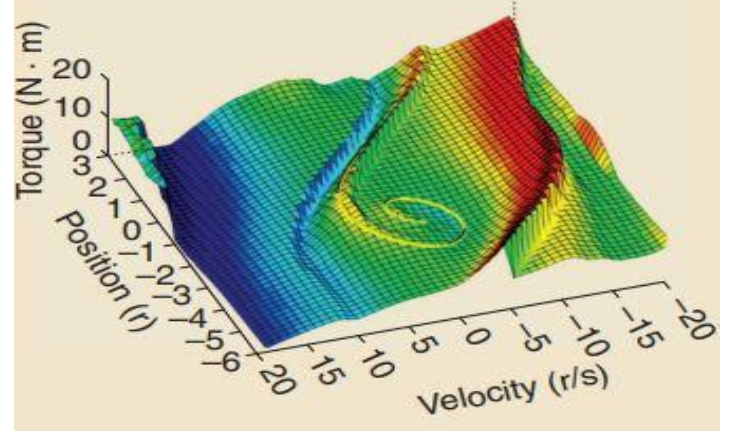

Figure 12 Different position vs torque condition and velocity 1

Figure 12 The $\mathrm{x}$-axis taken for velocity and $\mathrm{y}$ axis for position. Here the velocity peak value is $10.5 \mathrm{r} / \mathrm{s}$ and position is -2.5 and also torque is 20 was taken from this contour plot. These relations can be comprehended by studying the above plot.

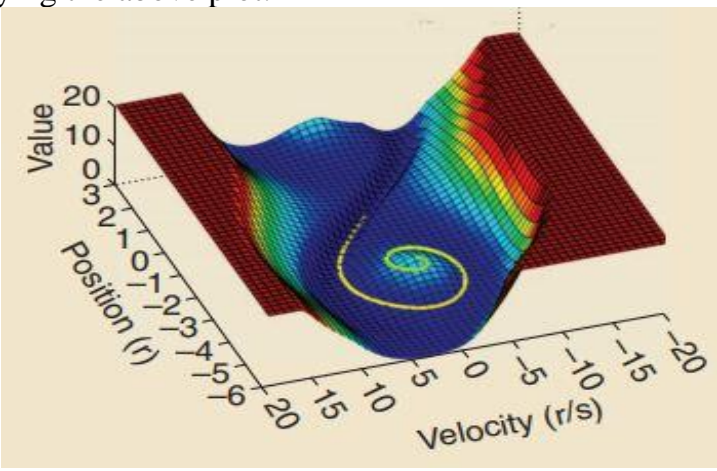

Figure 13 Different position vs torque condition and velocity 2
Figure 13 The $\mathrm{x}$-axis taken for velocity and $\mathrm{y}$ axis for position. Here the velocity peak value is $10.8 \mathrm{r} / \mathrm{s}$ and position is -2.5 and also torque is 20 was taken from this contour plot. The minimum values are taken the velocity is $2.3 \mathrm{~m} / \mathrm{s}$. These relations can be comprehended by studying the above plot.

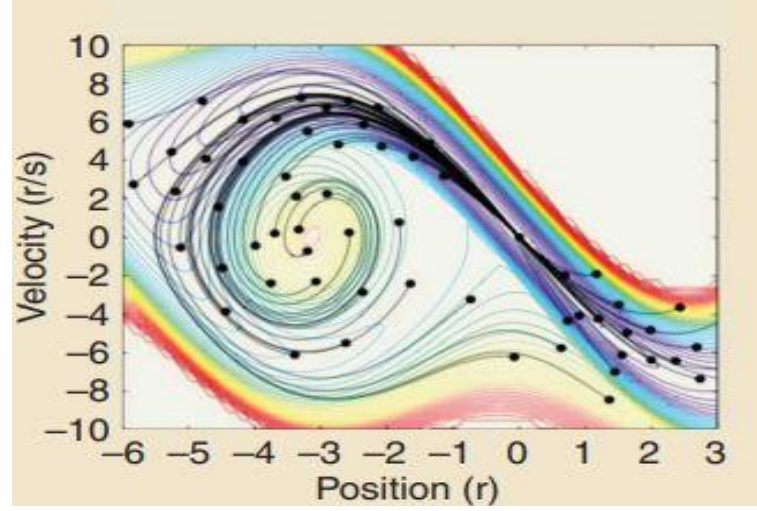

\section{Figure 14 Different position vs torque condition and velocity 3}

Figure 14 the $\mathrm{x}$-axis taken for position and $\mathrm{y}$ axis for velocity. Here the velocity peak value is $8.5 \mathrm{r} / \mathrm{s}$ and position are 2.5 was taken from this contour plot. So, the particular period of position the velocity and displacement were reduced. These relations can be comprehended by studying the above graph.

TABLE NO 2 COMPARISON OF SWING ANGLE, DISPLACEMENT AND TIME

\begin{tabular}{|c|c|c|c|c|c|}
\hline \multirow[t]{2}{*}{ s.no } & \multicolumn{2}{|c|}{ Swing angle $(\theta)$} & \multicolumn{2}{|c|}{$\begin{array}{l}\text { Displacement } \\
(\mathrm{mm})\end{array}$} & \multirow{2}{*}{$\begin{array}{c}\text { Tim } \\
\text { e } \\
\text { (sec } \\
\text { ) }\end{array}$} \\
\hline & $\begin{array}{l}\text { Existi } \\
\text { ng } \\
\text { model }\end{array}$ & $\begin{array}{l}\text { Propos } \\
\text { ed } \\
\text { model }\end{array}$ & $\begin{array}{l}\text { Existi } \\
\text { ng } \\
\text { model }\end{array}$ & $\begin{array}{l}\text { Propose } \\
\text { d model }\end{array}$ & \\
\hline 1 & 40 & 30 & 3 & 2.8 & 5 \\
\hline 2 & 40 & 30 & 2.7 & 2.4 & 10 \\
\hline 3 & 40 & 30 & 2.1 & 1.8 & 15 \\
\hline 4 & 40 & 30 & 1.9 & 1.5 & 20 \\
\hline
\end{tabular}

From the table no 2 the existing model swing angle is 40 and proposed model swing angle was reduced to the five degree. So, the displacement values are reduced the existing model to compare the proposed model. And also, the time period was taken for the 5 seconds. These relations can be comprehended by studying the above the table.

\section{CONClusion}

Pendulum was analyzed for the vibration in different angles. The velocity, acceleration, displacement and force. Now calculated from the numerical analysis for the existing reference pendulum and proposed pendulum. The proposed pendulum having less displacement, acceleration, velocity and force compare other. The Percentage of displacement reduced was 84 . Percentage of acceleration reduced was 82 .Percentage of force reduced was 81.66. This type of pendulum may be introduced in 


\section{Available online at www.ijrat.org}

reciprocating mechanism oriented automatic guided vehicle to reduce the vibration or better stability.

\section{REFERENCES}

[1] M.S.Alamand,M.O.Tokhi, 2007, "Design of a Command Shaper for Vibration Control of Flexible Systems a Genetic Algorithm Optimization Approach", journal of low frequency noise, vibration and active control, Pages $295-310$

[2] ZhongYi Chu and Jing Cui 2015. "Experiment on vibration control of a two-link flexible manipulator using an input shaper and adaptive positive position feedback", Advances in Mechanical Engineering Vol. 7(10) 1-13

[3] Bo Luo, Hai Huang, Jinjun Shan and Hidekazu Nishimura 2013,' Active vibration control of flexible manipulator using auto disturbance rejection and input shaping" journal of aerospace engineering, Vol. 228(10)

[4] Mohammad H. Fateh, S. Necsulescu and Ali A. Fatehi, 2019 , "Tracking control design for a multi-degree under actuated flexiblecable overhead crane system with large swing angle based on singular perturbation method and an energy-shaping technique" Journal of

\section{AUTHORS PROFILE}

C.Kirankumar, was completed his diploma in Mechanical Engineering in the year of 2014 and he also completed his B.E Mechanical Engineering in the year of 2017 and now he pursuing his M.E - Engineering Design second year in Government College of Technology, Coimbatore.

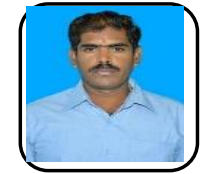

Dr.S.Periyasamy was currently working as Assistant Professor in the Department of Mech. Engg. Government College of Technology Coimbatore. He holds the educational qualifications of B.E - Mechanical Engineering, M.E - Aeronautical Engineering and Ph.D in Mechanical Engineering.

\section{Vibration and Control 0(0) 1-16}

[5] X. Zhao, Z. Zhang, J. Huang, Energy-based swing up control of rotary parallel inverted pendulum, in: Intelligent Control and Automation (WCICA), 2016 12th World Congress on, IEEE, 2016, pp. 666-671.

[6] X. Yang, X. Zheng, Swing up and stabilization control design for an underactuated rotary inverted pendulum system: theory and experiments, IEEE Trans. Ind. Electron. (2018).

[7] V. Casanova, J. Alcaína, J. Salt, R. Pizá, Á. Cuenca, Control of the rotary inverted pendulum through threshold-based communication, ISA Trans. (2015). 